\title{
Insight into the intestinal microbiome of farrowing sows following the administration of garlic (Allium sativum) extract and probiotic bacteria cultures under farming conditions
}

\author{
Marta Satora ${ }^{1}$, Marcin Magdziarz², Anna Rząsa ${ }^{3}$, Krzysztof Rypuła $^{1}$ and Katarzyna Płoneczka-Janeczko ${ }^{1 *}$ (D)
}

\begin{abstract}
Background: Due to the tendency to reduce antibiotic use in humans and animals, more attention is paid to feed additives as their replacement. Crucial role of feed additives is to improve the health status, production efficiency and performance. In this original research, we estimate the potential influence of garlic (Allium sativum) extract and probiotic formula including Enterococcus faecium, Lactobacillus rhamnosus and Lactobacillus fermentum on the intestinal microbiota of sows, using the next generation sequencing method (NGS).

Results: Our results indicate that the overall species richness as well as the composition of swine gut microbiota may be shaped by regular feeding with supplemented additives. On the Family and Genus level both additives (garlic extract and probiotics) seem to decrease microbiome diversity and richness. However, when it comes to garlic supplementation, we found the opposite trend on the Species level.

Conclusions: The analysis of the selected microbial function indicates that both additives used in this study (garlic extract and composition of probiotics) seem to create a greater metabolic potential than estimated in a control group of sows. A general trend of losing or decreasing members of pathogenic species in the swine microbiome seems to occur in relation to both supplemented additives. In the prevention of some bacterial diseases supplemented additives could be considered for future use.
\end{abstract}

Keywords: Swine microbiome, Shaping, Garlic, Lactobacillaceae, Nutrition

\section{Background}

Over the last decade, the majority of scientists have been involved in research concerning the swine microbiome using differences in the bacterial $16 \mathrm{~S}$ rRNA gene sequence and the next generation sequencing method (NGS). Isaacon and Kim presented a review of swine

\footnotetext{
* Correspondence: katarzyna.ploneczka-janeczko@upwr.edu.pl

'Department of Epizootiology with Clinic for Birds and Exotic Animals,

Faculty of Veterinary Medicine, Wrocław University of Environmental and Life Sciences, Plac Grunwaldzki 45, Wrocław, Poland

Full list of author information is available at the end of the article
}

gastrointestinal (GI) microbiome, documenting a close relationship between the development of the microbiome and the sampling place within the GI tract as well as the growth of animals [1]. It has been confirmed in several studies that many agents have an influence on swine gut colonization by microorganisms starting from birth. They stem from the sows' vagina, skin and faeces microbiome, through diet components, environment, infections and stress factors. Gastrointestinal microbiota constitute a dynamic structure which may evolve over

C C The Author(s). 2020 Open Access This article is licensed under a Creative Commons Attribution 4.0 International License, which permits use, sharing, adaptation, distribution and reproduction in any medium or format, as long as you give appropriate credit to the original author(s) and the source, provide a link to the Creative Commons licence, and indicate if changes were made. The images or other third party material in this article are included in the article's Creative Commons licence, unless indicated otherwise in a credit line to the material. If material is not included in the article's Creative Commons licence and your intended use is not permitted by statutory regulation or exceeds the permitted use, you will need to obtain permission directly from the copyright holder. To view a copy of this licence, visit http://creativecommons.org/licenses/by/4.0/ The Creative Commons Public Domain Dedication waiver (http://creativecommons.org/publicdomain/zero/1.0/) applies to the data made available in this article, unless otherwise stated in a credit line to the data. 
time [2-4]. Holman et al. [5] prepared a meta-analysis report, based on previously collected data. Mentioned authors concluded that the results of the different studies on swine gut microbiota needed to be optimized, regardless of the country or place where the sample was taken (i.e. various parts of the gastrointestinal tract, where the samples were collected). The presence of yeast or viruses in the faecal samples of pigs was also studied using a culture-independent method [6, 7].

Taking into account health requirements and consumer protection, the development of antimicrobial resistance and the potential transfer of bacterial genes responsible for bacterial resistance between animals and humans as well as the possible environmental contamination, according to the implementation of EU legislation - antibiotics as growth promoters (AGPs) in animal feed have been banned from animal production in EU countries based on EC Regulation No. 1831/2003 [8, 9]. An issue of common concern among owners and veterinarians who are directly engaged in the economics of pig production as well as scientific researchers is therefore the possibility of implementing alternative substances that could have an influence on the efficient production of livestock. New feed additives have been developed, which are authorised in accordance with the same regulation (EC 1831/2003) as well as with Regulation No. $767 / 2009$ of the European Parliament and of the Council regulates feed availability on the market and the use of feed, amending European Parliament and Council Regulation (EC) No. 1831/2003 and repealing Council Directive 79/373/EEC, Commission Directive 80/511/EEC, Council Directives 82/471/EEC, 83/228/EEC, 93/74/ EEC, 93/113/EC and 96/25/EC and Commission Decision 2004/217/EC. With reference to the EC definition [10]: the role of feed additives used in animal nutrition is to improve the quality of feed, quality of food of animal origin or to improve an animal's performance and health e.g. providing enhanced digestibility of feed components, correct its appearance, taste, smell or consistency. Therefore, the crucial role of the feed additives is to improve health status, production efficiency and performance. Among this group live probiotic bacteria cultures, prebiotics and synbiotics, acidifiers, feed enzymes, aromatic and flavouring agents, preservatives, antioxidants or yeast products could be found [11-14]. Their role is attributed to modulate or protect the immune system of pigs and participate in the regulation of the gut microbiota composition. Compounds isolated from plants also enjoy a great interest among feed additives (phytogenic feed additives, PFA) containing biologically active substances $[15,16]$. One of them is garlic (Allium sativum) extract. The suitability of garlicderived compounds has been evaluated in recent years in different species [17-22]. It has been shown that in broilers, the introduction of the garlic extract supplement has statistically significant potential to improve body weight gain in the first 4 weeks of life, compared with the control group supplemented with ciprofloxacin [20]. Based on the review paper by Senthilkumar et al. which includes numerous studies on the effectiveness of garlic extract, it could be concluded that garlic may enhance the feed conversion ratio (FCR) of these birds as well as decrease the mortality rate [21]. Supplementation of garlic may also influence the immunity of broilers, by increasing expression (mRNA) of the Toll-like receptors (TLR2, TLR4 and TLR7) and thus also make it possible to modulate the T-cell mediated immune response with garlic [23]. It has been confirmed that garlic or its derivatives can enhance the cellular and humoral immune system by stimulating some cells (e.g. macrophages, lymphocytes, NK) or mechanisms (e.g. immunoglobulin E production). It exhibits beneficial effects against microbial infections and anti-inflammatory activity [24] but its influence on production parameters is controversial. It was reported that garlic extract improves growth performance, nutrient digestibility and meat quality in pigs [25], however other authors did not observe such effects [26]. A garlic additive or its derivatives seem to be an interesting alternative to antibiotics. Tatara et al. confirmed that such additives facilitate good health status, performance and the systemic development of piglets [17].

Also, the use of probiotic bacteria as a feed additive for piglets and sows has been evaluated in recent years [27-29]. It is well documented that probiotics can reduce digestive disorders and improve productive parameters. They are especially recommended for piglets because of their rapid growth, immature immune system, digestive capacities and unstable intestinal microbiota. The largest group satisfying the definition of probiotics proposed by the International Scientific Association for probiotics and prebiotics (2013), are bacteria belonging to the Genera Bacillus, Enterococcus, Lactobacillus, Pediococcus and Streptococcus. In the European Union the QPC concept for probiotics (Qualified Presumption of Safety, including a history of safe usage and the absence of the risk of acquired resistance to antibiotics) has also been postulated [30]. The effects of probiotics in swine production has been evaluated in numerous publications. They boost the microbial composition in the gut and modulate the immune status. Moreover, they improve the average daily gain (ADG), average daily feed intake (ADFI) and feed conversion ratio (FCR), but there are still some uncertainties concerning their usage [31, 32]. These are mainly associated with the health status and maturity of various animals, farm conditions and kind of strains and proportions between them [11, 29, 33]. This dependency is not always 
identified and predictable which has prompted a search for new strategies and solutions. A bacterial strain of Enterococcus faecium was previously described in a study conducted by Napiorkówska et al. [34] where it was used in piglets and its impact on the composition of faecal microbiota was evaluated. The studies showed that the use of a probiotic compound in piglets had a positive impact on daily weight gain as well as a reduction of diarrhoea incidences. Piglets supplemented with Enterococcus faecium had an increased number of bacteria from the Enterobacteriaceae family, Lactobacillus spp., Lactococcus spp., and S. cerevisiae yeast were found. A reduction of diarrhoea incidences after the oral administration of a Enterococcus faecium supplement has also been observed by Zeyner and Boldt [35]. The adhesive capability of Lactobacillus fermenti 126 and Lactobacillus rhamnosus CCM 1825 has been evaluated by Brzozowski et al. [36]. It was concluded, that the analysed strains of lactic acid bacteria synthetize biosurfactant, whose molecules consist of proteins, polysaccharides and phosphates, which demonstrate good anti-adhesive properties against Enterobacteriaceae. Lactobacillus rhamnosus was also effective in ameliorating diarrhoea in weaned piglets [37]. Another study evaluated Lactobacillus fermentum effects on piglets' growth, faecal microbiota, immune index and antioxidant activity [38]. Administration of the described probiotic bacteria increased the weight gain of piglets during the first 2 weeks of life, increased the number of Lactobacillus in faeces and increased the concentration of IgM, IgG, IgA as well as the activity of glutathione peroxidase in the plasma of the piglets.

Based on the literature review from the metaanalysis performed by Holman et al. [5] feed additives such as amylase and amylopectin, calcium phosphorus, distillers' dried grains or resistant starch have been analysed and their influence on the gut microbiota has already been tested in the swine. The aim of this study was to investigate the potential influence of a garlic (Allium sativum) extract and probiotic formula including Enterococcus faecium, Lactobacillus rhamnosus and Lactobacillus fermentum on the intestinal microbiota of sows.

\section{Results}

\section{DNA sequence data}

A total of 5,738,676 DNA-identified sequences that were obtained in this study were then subsequently analysed in the metagenomic classification of the microbiota of farrowing sows, from the Kingdom to the Species taxonomic level. The DNA sequences were analysed for all individuals within groups, which was possible thanks to an individual bar code identifier for each pig sample. Table 1 illustrates the details regarding the number of
Table 1 Identified sequences for sows (A1-A8, B1-B8, C1-C8) and groups $(A, B, C)$

\begin{tabular}{|c|c|c|c|c|c|}
\hline & Kingdom & Phylum & Family & Genus & Species \\
\hline A1 & 27,246 & 26,609 & 24,920 & 24,455 & 13,648 \\
\hline A2 & 51,743 & 50,301 & 48,427 & 47,154 & 24,260 \\
\hline A3 & 49,218 & 48,271 & 46,663 & 45,712 & 27,148 \\
\hline A4 & 59,851 & 59,109 & 57,914 & 56,719 & 31,565 \\
\hline A5 & 54,671 & 53,934 & 52,103 & 50,945 & 30,475 \\
\hline A6 & 50,797 & 50,071 & 48,868 & 47,654 & 27,182 \\
\hline A7 & 62,604 & 61,989 & 60,309 & 58,953 & 30,083 \\
\hline A8 & 48,433 & 47,794 & 46,771 & 45,873 & 29,148 \\
\hline \multicolumn{6}{|l|}{ Group A } \\
\hline Total & 404,563 & 398,078 & 385,975 & 377,465 & 213,509 \\
\hline Mean & $50,570.375$ & $49,759.75$ & $48,246.88$ & $47,183.13$ & $26,688.63$ \\
\hline SD & $10,687.7996$ & $10,669.15$ & $10,710.92$ & $10,449.1$ & 5758.181 \\
\hline B1 & 45,228 & 43,994 & 42,998 & 42,145 & 25,702 \\
\hline B2 & 108,414 & 106,150 & 103,524 & 101,310 & 52,313 \\
\hline B3 & 54,364 & 53,245 & 51,720 & 50,385 & 27,647 \\
\hline B4 & 59,115 & 57,891 & 56,009 & 54,638 & 28,518 \\
\hline B5 & 50,822 & 50,265 & 47,912 & 46,849 & 24,228 \\
\hline B6 & 57,400 & 56,846 & 53,653 & 52,548 & 27,342 \\
\hline B7 & 47,846 & 47,299 & 45,401 & 44,333 & 26,100 \\
\hline B8 & 46,936 & 46,299 & 43,581 & 41,531 & 22,325 \\
\hline \multicolumn{6}{|l|}{ Group B } \\
\hline Total & 470,125 & 461,989 & 444,798 & 433,739 & 234,175 \\
\hline Mean & $58,765.625$ & $57,748.63$ & $55,599.75$ & $54,217.38$ & $29,271.88$ \\
\hline SD & 20,673.8936 & $20,175.28$ & $19,936.02$ & $19,617.94$ & 9519.386 \\
\hline C1 & 57,695 & 56,221 & 53,479 & 52,223 & 28,243 \\
\hline C2 & 56,074 & 54,901 & 52,329 & 51,092 & 28,208 \\
\hline C3 & 61,661 & 60,692 & 59,245 & 57,684 & 27,546 \\
\hline C4 & 51,604 & 50,888 & 49,434 & 48,222 & 23,518 \\
\hline C5 & 61,213 & 60,729 & 55,946 & 54,754 & 31,255 \\
\hline C6 & 53,043 & 51,956 & 50,081 & 48,859 & 22,865 \\
\hline C7 & 49,755 & 48,949 & 47,359 & 46,107 & 23,856 \\
\hline C8 & 51,750 & 50,613 & 48,270 & 47,236 & 28,705 \\
\hline \multicolumn{6}{|l|}{ Group C } \\
\hline Total & 442,795 & 434,949 & 416,143 & 406,177 & 214,196 \\
\hline Mean & $55,349.375$ & $54,368.63$ & $52,017.88$ & $50,772.13$ & $26,774.5$ \\
\hline SD & 4533.13953 & 4560.18 & 4072.079 & 3968.941 & 3000.356 \\
\hline
\end{tabular}

identified sequences for individual sows and for the groups.

\section{Microbial diversity}

The microbial diversity of farrowing sows after garlic or probiotic bacteria administration in all eight pigs in each group and the control group was measured using 
Shannon and Simpson diversity indices. The next Figs. $1,2,3$ present the obtained results for each available taxonomic level (Family, Genus and Species).

In Table 2, details of the diversity measures (the average Shannon and Simpson diversity indices within groups) for the Family, Genus and Species the taxonomic levels are included.

The comparison of microbial diversity takes into account the Family and Genus taxonomic levels highlighted in the same trend - the most heterogeneous bacteria composition was estimated to be in control group $C$ and the lowest diversities were observed in the group supplemented with probiotic bacteria (B). However, this tendency changed on the Species level - the highest microbial diversity was observed after the administration of garlic extract (group A).

\section{Taxon-dependent analysis}

A taxon-dependent analysis of the obtained results was performed to demonstrate possible shifts in the composition of faecal microbiota of sows administered with garlic extract and the composition of probiotic bacteria when compared to the control group. The results of the Kingdom distribution (Table 3) demonstrated that the proportion of Bacteria was almost identical in group A (92.64\%) and C (92.89\%) and slightly lower in the sows of group B that were supplemented with probiotics (91.03\%).

A contrary tendency was visible, one that took into account the participation of Archaea - the largest proportion was in group B (2.99\%) if compared with A (1.61\%) or C $(1.36 \%)$. For the needs of this study, at the subsequent taxonomic identification levels (Phylum, Family,
Genus and Species) more than 1\% of the total identified DNA sequences at least in one of the individuals was established as "abundant" as previously proposed by Kim et al. [39]. Several differences in the abundance of bacterial taxa between sows supplemented with garlic extract and a composition of probiotic bacteria were detected. Figure 4 shows the detailed distribution of abundant microorganisms on the Phylum taxonomic rank.

Two Families: Clostridiaceae (47.09, 48.04 and $39.02 \%$ in A, B, C respectively) and Turicibacteriaceae $(6.84,7.34$ and $10.56 \%$ as previously) were mostly identified within all sow groups. The proportion of sequences that could not be assigned to the Family taxon was $4.89,5.53$ and $6.0 \%$ in the group A, B and C respectively. The other Families identified in proportion $\leq 1 \%$ in the same groups were 4.62, 4.09 and $4.58 \%$. A total of 30 bacterial Families have been identified. Considering the differences, where at least in one of the sows within the Family taxon group could be identified as abundant $(>1 \%) 26,19$ and 23 Families were present for the A, B and C groups. For all three groups Actinomycetacea, Clostridiaceae, Coriobacteriaceae, Corynebacteriaceae, Fusobacteriaceae, Lachnospiraceae, Lactobacillaceae, Methanobacteriaceae, Moraxellaceae, Peptococcaceae, Peptostreptococcaceae, Prevotellaceae, Ruminococcaceae, Streptococcaceae, Thermicanaceae and Thuricibacteriaceae have been identified. In the sows supplemented with garlic extract in comparison with control groups, Aerococcaceae, Bifidobacteriaceae, Comamonadaceae, Dietziaceae, Flavobacteriaceae, Methylabacteriaceae and Staphylococcaceae have been shown. When comparing Families representative for sows supplemented with probiotic and control groups, the difference was recognised for the Methylacidibacteriaceae only, that were absent from the control group.

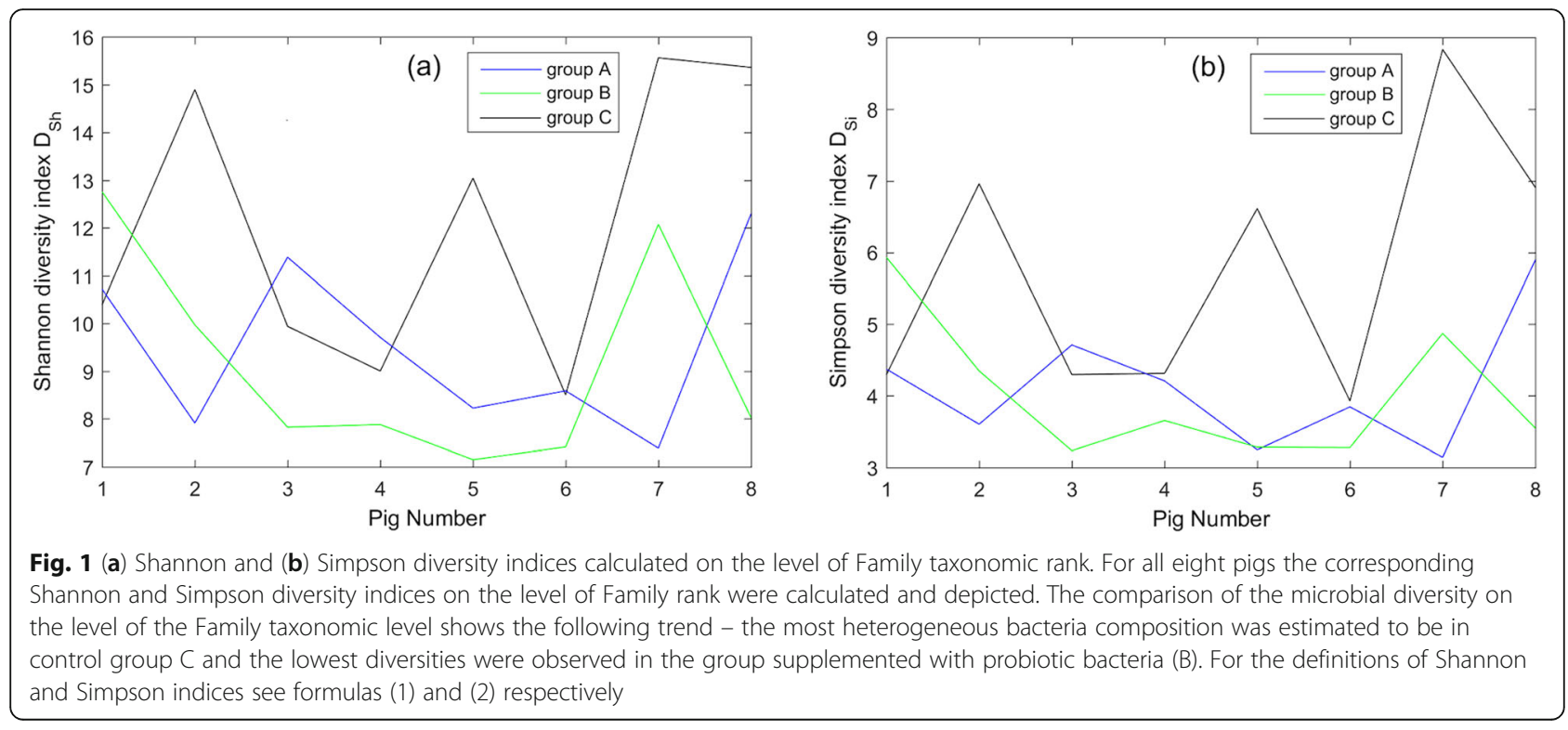



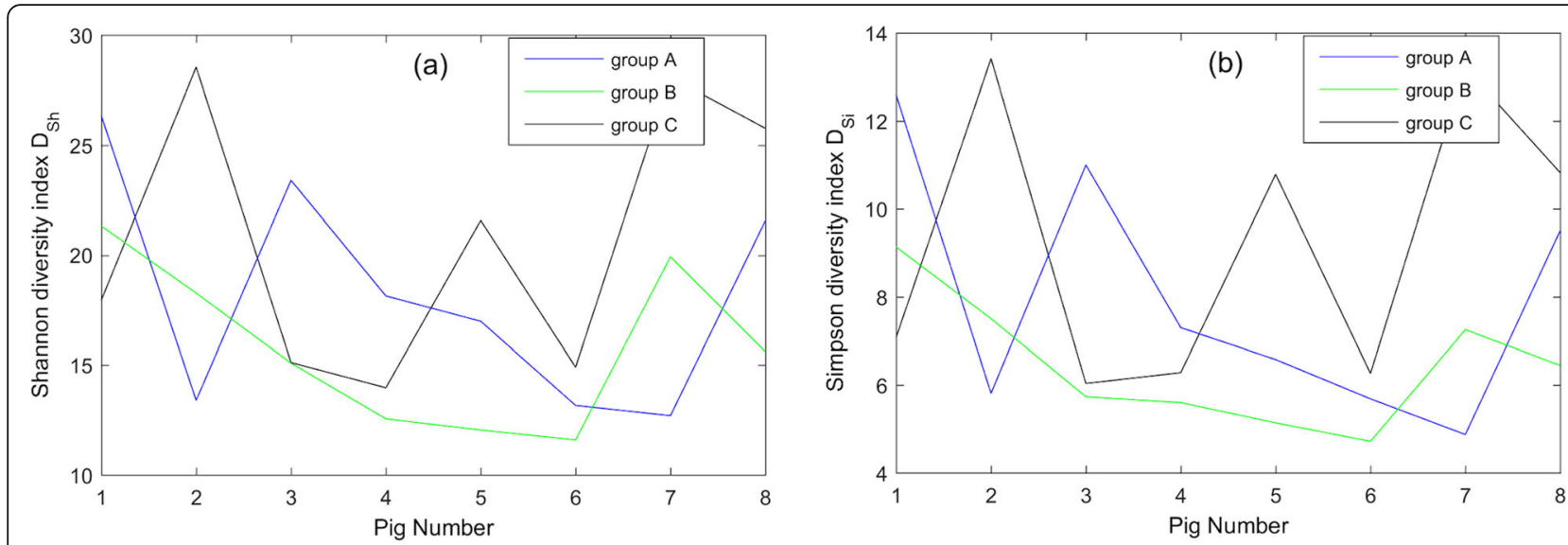

Fig. 2 (a) Shannon and (b) Simpson diversity indices calculated on the level of Genus taxonomic rank. For all eight pigs the corresponding Shannon and Simpson diversity indices on the level of Genus rank were calculated and depicted. The comparison of the microbial diversity on the level of the Genus taxonomic level shows the following trend - the most heterogeneous bacteria composition was estimated to be in control group C and the lowest diversities were observed in the group supplemented with probiotic bacteria (B). For the definitions of Shannon and Simpson indices see formulas (1) and (2) respectively

Supplementation of garlic extract as well as probiotics may promote the absence of Campylobacteraceae and Heliobacteraceae. Garlic may also influence the absence of Erysipelotrichaceae and Veilonellaceae, that were recognised in the sows supplemented with probiotics and in the control groups. In sows fed with probiotics Bacteroidaceae, Enterobacteriaceae and Porphyromonadaceae have not been identified, although they were present in group A as well as C. Figure 5 shows detailed characteristics of microbiota within groups A, B and C on the level of Family rank.

Clostridium (31.89\% in A, $35.65 \%$ in B and $28.75 \%$ in C) as well as Turicibacter $(6.84 \%$ in $\mathrm{A}, 7.33 \%$ in B and $10.56 \%$ in C) were the most abundant Genera within all the groups. The proportion of sequences that could not be assigned to the Genus rank was 6.97, 7.94 and 8.25\% in the groups A, B and C. The other Genera identified in proportion $\leq 1 \%$ in the same groups were $7.88,7.21$ and $7.80 \%$. A total of 45 Genera have been identified in this study; 34 in group A, 23 in B and 37 in $C$ respectively. For all three groups: Acinetobacter, Actinomyces, Alcaliphilus, Anaerococcus, Arcanobacterium, Blautia, Clostridium, Corynebacterium, Fusobacterium, Lactobacillus, Methanobrevibacter, Mogibacterium, Oscillospira, Peptoniphilus, Prevotella, Psychrobacter, Ruminococcus, Sarcina, Streptococcus, Thermicanus and Turicibacter were identified. In the sows supplemented with garlic extract
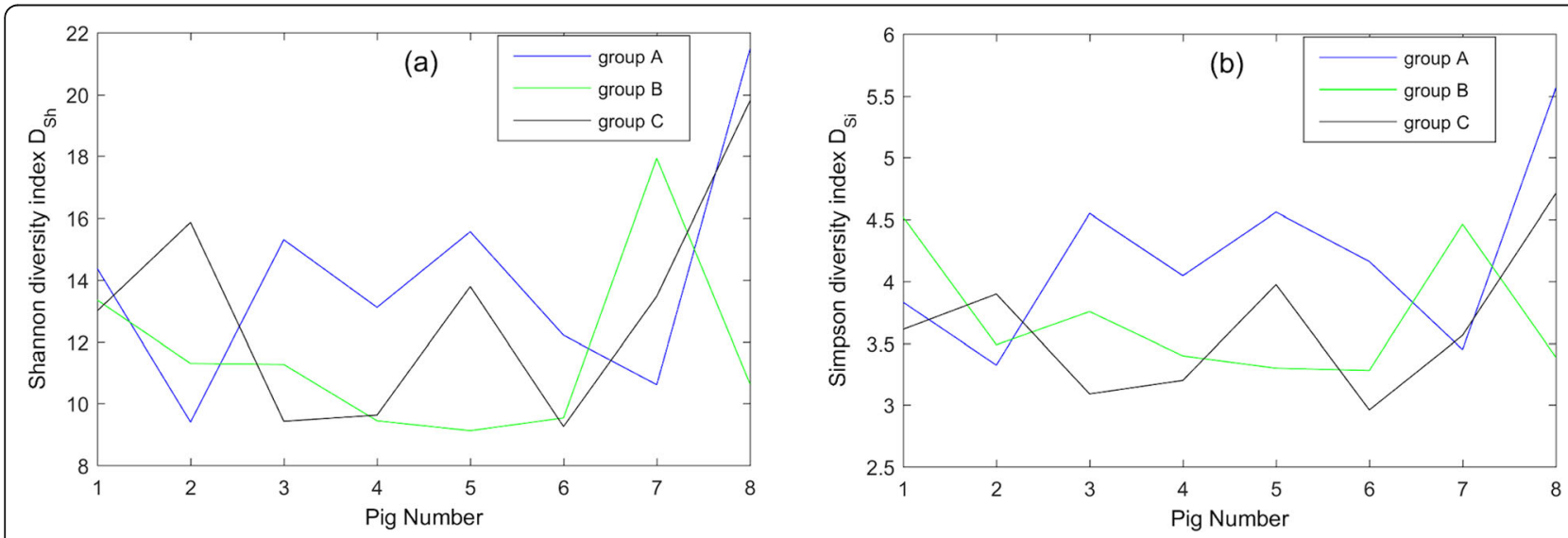

Fig. 3 (a) Shannon and (b) Simpson diversity indices calculated on the level of Species taxonomic rank. For all eight pigs the corresponding Shannon and Simpson diversity indices on the level of Species rank were calculated and depicted. The tendency observed in Figs. 1 and 2 changed on the Species level - the highest microbial diversity was observed after the administration of the garlic extract (group A). For the definitions of Shannon and Simpson indices see formulas (1) and (2) respectively 
Table 2 Comparison of microbial diversity between groups $(A, B)$ in relation to the control group (C)

\begin{tabular}{|c|c|c|c|c|c|c|}
\hline & \multicolumn{2}{|l|}{ Family } & \multicolumn{2}{|l|}{ Genus } & \multicolumn{2}{|l|}{ Species } \\
\hline & Shannon index ${ }^{a}$ & Simpson Index ${ }^{a}$ & Shannon index & Simpson index & Shannon index & Simpson index \\
\hline Group A & 9.5278 & 4.1271 & 18.2079 & 7.9176 & 14.0041 & 4.1848 \\
\hline Group B & 9.1371 & 4.0175 & 15.8000 & 6.4409 & 11.5741 & 3.6983 \\
\hline Group C & 12.0881 & 5.7677 & 20.7367 & 9.2367 & 13.0290 & 3.6255 \\
\hline
\end{tabular}

${ }^{a}$ For the definitions of Shannon and Simpson indices see formulas (1) and (2) respectively

in comparison with control groups, Aerococcus, Bifidobacterium, Cetobacterium, Comamonas, Dietzia and Dysgonomonas were detected. Garlic seems to support the presence of these Genera because they have also not been demonstrated in the sows supplemented with probiotics. However, when comparing Genera representative of control sows and the probiotic group, there was no characteristic Genus identified with regard to probiotics only. Both additives seem to promote Coloramator and Candidatus Methylacidofilum, Genera that were absent from the control group.

In sows that had been fed with garlic extract as well as with probiotics Bulleidia, Campylobacter, Collinsella, Eubacterium, Faecalibacterium, Heliorestis, Mobiluncus, Natronincola, Olsenella, Oribacterium and Slackia were not identified. Additionally, Bacteroides, Escherichia, Peptococcus, Peptostreptococcus and Porphyromonas were absent in sows from group B, although they were present in group A as well as $\mathrm{C}$.

Figure 6 shows the detailed characteristics of microbiota within group A, B, C on the level of Genus rank.

Clostridium cadaveris (10.77\% in A, $12.70 \%$ in B and $8.65 \%$ in C), Alcaliphilus crotonatoxidans (4.30\% in A, $5.02 \%$ in B and $3.89 \%$ in C), Clostridium butyricum (2.21\% in A, $2.15 \%$ in B and $1.63 \%$ in C), Sarcina maxima $(2.18 \%$ in $\mathrm{A}, 2.11 \%$ in $\mathrm{B}$ and $1.81 \%$ in $\mathrm{C}$ ) as well as Lactobacillus ultunensis $(1.73 \%$ in $\mathrm{A}, 1.78 \%$ in $\mathrm{B}$ and $1.27 \%$ in $C)$ and Turicibacter sanguinis $(1.71 \%$ in A, $1.72 \%$ in $\mathrm{B}$ and $2.47 \%$ in $\mathrm{C}$ ) were the most abundant Species within all three groups. The proportion of sequences that could not be assigned to the Species rank was $47.21,49.81$ and $51.62 \%$ in the group A, B and C. The other Genera identified in proportion $\leq 1 \%$ in the same groups were $14.26,13.04$ and $15.41 \%$. A total of 43 Species have been identified in this study; 32 within group A, 19 in B and 29 in C, respectively. There were no differences between all three groups when it comes

Table 3 Relative abundance of microorganisms on the Kingdom level in sows group A, B and C

\begin{tabular}{lllll}
\hline & Bacteria & Archeae & Unclassified & Other \\
\hline Group A & $92.4 \%$ & $1.61 \%$ & $4.29 \%$ & $0.81 \%$ \\
Group B & $91.03 \%$ & $2.99 \%$ & $4.93 \%$ & $0.62 \%$ \\
Group C & $92.89 \%$ & $1.36 \%$ & $4.94 \%$ & $0.79 \%$ \\
\hline
\end{tabular}

to the presence of Acinetobacter lwofii, Actinomyces hyovaginalis, Alcaliphilus crotonatoxidans, Alcaliphilus peptidifermentans, Arcanobacterium pluranimalium, Clostridium butyricum, Clostridium cadaveris, Corynebacterium puruviciproducens, Corynebacterium xerosis, Lactobacillus ultunensis, Prevotella buccalis, Sarcina maxima, Streptococcus bovis and Thuricibacter sanguinis. Garlic extract in comparison with control groups seems to support the presence of Acinetobacter indicus, Aerococcus sanguinicola, Bacteroides sartorii, Bifidobacterium pseudolongum, Cetobacterium ceti, Clostridium saccharoperbutylacetonicum, Corynebacterium hansenii, Psychrobacter halophilus and Psychrobacter proteolyticus, as these bacteria were also absent from the group fed with probiotics. When comparing Species representative for the probiotic group and control sows, probiotics may have an influence on the presence of Lactobacillus antri and Lactobacillus reuteri, which were absent as well from the sows supplemented with garlic extract.

Both additives seem to promote the occurrence of Caloramator mitchellensis, Lactobacillus pontis and Ruminococcus bromii that were undetected in group C.

In sows fed with garlic extract as well as probiotics Bacteroides fragilis, Colinsella aerofaciens, Corynebectarium amycolatum, Eubacterium biforme, Methanobrevibacter smithii, Olsenella uli, Oribacterium sinus, Prevotella copri, and Streptococcus suis were not identified. Additionally, in sows fed with probiotics Anaerococcus octavius and Anaerococcus tetradius, Peptoniphilus gorbachii, Peptostreptococcus anaerobius, and Porphyromonas endodonthalis and Porphyromonas somerae were absent, although they were present in group A as well as in C. Figure 7 shows the detailed characteristics of microbiota within group A, B, C on the level of Species rank.

\section{Discussion}

From the point of view of swine production (including both economic and health status of the animals) beneficial effects would have been apparent if modulation of the microbiome had led to the restriction of microorganisms, generally considered as pathogens or conditionally pathogenic. It would also be desirable to obtain such amendments in the microbiome. This would allow for 


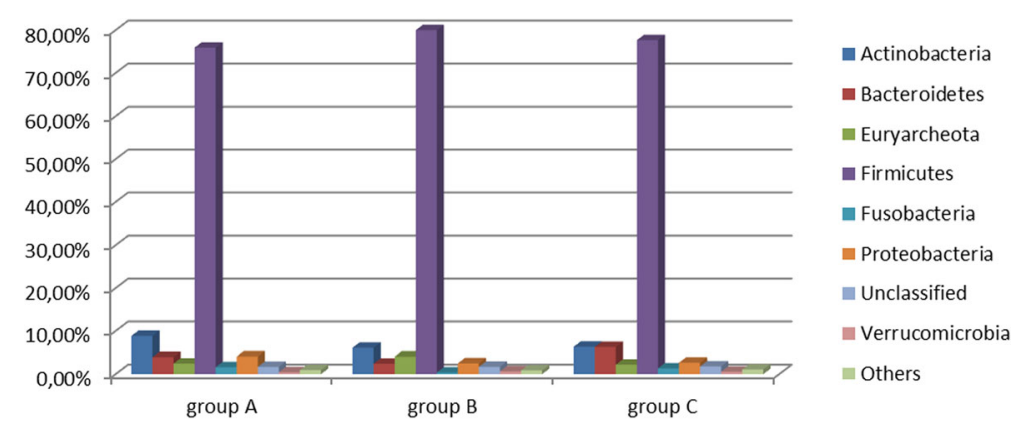

Fig. 4 The mean relative abundance (\%) of identified sequences on the Phylum level. The figure shows the detailed distribution of abundant microorganisms on the Phylum taxonomic rank. We observe that the proportion of Firmicutes in sows supplemented with garlic is slightly lower than in controlled pigs, however a significant increase in this group of microorganisms after probiotic supplementation.

better utilization of nutrients as well as a favourable energy balance that took into account metabolic processes.

Comparing our own results with those reported in other studies on pigs [40] the same tendency of high diversity in the microbial communities appeared. Differentiation in some taxa between experimental groups seem to have been connected with investigated additives which influenced the gastrointestinal microbiota; moreover, individual variability in the frame of the experimental groups was observed. It was also observed that the diversity and richness of the microbial community underwent a change together with the taxonomic level of identification. Some kinds of bacteria that were identified on the Family and Genus level, disappeared on the Species level. On the Family and Genus level both additives (garlic extract and probiotics) seem to decrease microbiome diversity and richness, however we found an opposite trend on the species level, taking into account garlic supplementation. Wide variations between presented taxonomic and functional shifts in the pigs' gut microbiome is evident, based on the literature data. In the study by Homan et al. [5] among at least $90 \%$ of gastrointestinal samples, regardless of conditions, the genus Clostridium, Blautia, Lactobacillus, Prevotella, Ruminococcus, Roseburia, the RC9 gut group and Subdoligranulum were found. According to the authors cited above, these bacteria may serve as markers of a typical swine gut microbiota. Our results are in accordance with a large part of marked microorganisms, although Genus Roseburia or Genus Subdoligranulum have not been identified.

Quan et al. [41] compared gut microbiota of healthy fatteners which differed in daily gains, which resulted in a differentiation of FCR. Looking for potential relationships with FCR, functional profiles of gut microbiota seem to have appeared. Two digestion pathways, including carbohydrate metabolism (fermentation) and lipid metabolism were mainly represented in the colon. In the previous study on microbiome it was shown that the metabolism of Archaea is characterised by the absence of many classic routs, especially in carbohydrate metabolism - by which the degradation of some components differs strongly from typical pathways established by bacteria [42]. Archaea may also be involved in the methanogenesis (methane production by intestinal representatives) and different mechanisms of energy conservation, which could be beneficial in some conditions [43, 44]. Moreover, some studies have also demonstrated that Archaea seems to be a promising pharmabiotic, that can provide a supporting role even in the treatment of certain diseases, similarly to probiotics $[45,46]$. Analysis of our data revealed that the supplementation of probiotics may have an effect on the increasing proportion of $\mathrm{Ar}$ chaea in the pig's gastrointestinal microbiome, thus promoting the functional composition of the metagenome reshaped by diet additives. Bacteria that could be involved in metabolical pathways include Prevotella spp., Clostridium, Blautia and Ruminococcus, among others [5]. Bacteria in the genus Ruminococcus belong to important microbial symbionts, that have been found in numerous mammals, including pigs. The majority of well-known ruminococci utilise in their metabolism carbohydrates, including cellulitic and non-cellulitic species [47]. In our study, we found agreement between probiotic supplementation and the presence of Ruminococcaceae from the Family to the Species taxonomic level and identified species Ruminococcus bromii may facilitate the utilisation of resistant starches [48]. Recent studies have shown that an increased abundance of Prevotella may improve glucose metabolism, especially if the diet is enriched by fibre [49]. Changes in the abundance of Prevotella sp. were noted in pigs in response to diet, where during the nursing period and milk-oriented microbiome - there was only a small population of described taxa [50]. Exposure to garlic extract or probiotics in this study, did not enhance the prevalence of 


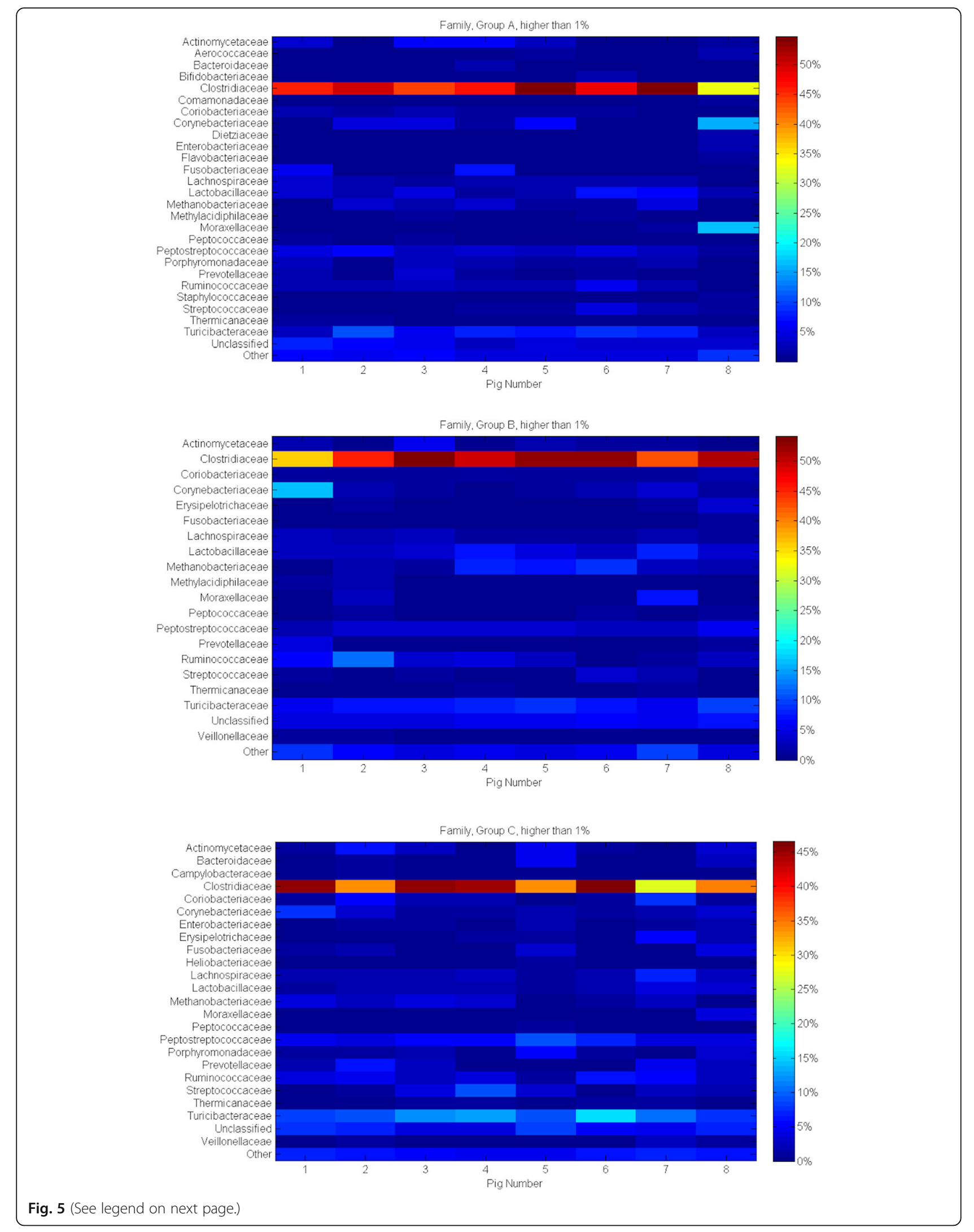


(See figure on previous page.)

Fig. 5 Prevalent Families analysis heat-maps of pig faecal microbiota considering feed additives and individual animals. The heat-map shows the relative abundance of identified sequences between individuals within the examined groups on the Family level. Density and saturation of each colour identify percentage (\%) of sequences specific for analysed taxonomic rank. Presented are only sequences exceeding $1 \%$. As 'Other' were marked the remaining sequences identified on the level lower than $1 \%$

Prevotella sp. Additionally, the highest percentage of identification was observed in the control sows at every taxonomic level (Prevotellaceae, Prevotella, Prevotella buccalis and copri). Another microorganism that may provide energy from polisascharides that other gut microorganisms cannot decompose is Blautia (within the family Lachnospiraceae) [51]. In this study, garlic extract and probiotics did not show any modulatory effect on the prevalence of the genus Blautia. We also found that sequences characteristic of Blautia were not identified on the species level.

In recent years, attention has been paid to butyrate production with reference to its role in the maintenance of gut homeostasis and epithelial integrity and alternative ways of producing them need to be taken into account [52]. Clostridiaceae are an important group of bacteria for humans and animals, covering both commensal and pathogenic species. This family plays a significant role in the metabolic welfare of colonocytes, for which butyrate is an essential energy source. Alternative butyrate-producing pathways have been found in Fusobacteria and Bacteroidetes [52]. In our study, 59 Clostridium spp. were identified, whereas among abundant (>1\%) species, two were confirmed - Clostridium butyricum and Clostridium cadaveris. In both cases, the occurrence of Clostridium butyricum as well as Clostridium cadaveris increased with garlic supplementation as well as with probiotic additives, compared to the control group. The same tendency was visible on the genus level. Clostridium butyricum is human and animal common gut commensal bacterium, however in recent years particular attention has been paid to the role of this species - both beneficial and pathogenic [53]. Positive properties of this bacterium include its involvement in the production of high amounts of butyric acid, interleukin 1 and prevention of the development of acute colitis with the inflammation of the mucous membrane. However some strains of C. butyricum investigated in humans may be implicated in pathological conditions, including necrotizing changes [53]. Clostridium cadaveris is a commensal bacterium, selectively pathogenic in immunocompromised individuals [54]. Although several Clostridia are involved in the development of infectious diseases due to the production of toxins (Clostridium perfringens, Clostridium difficle), in the presented study, Clostridia considered to be pathogenic were identified as being not abundant (below 1\% of the confirmed sequences for all groups). The metabolic activity of sows is also influenced by the occurrence in the methanogenic environment of Alcaliphilus crotonatoxidans that participate in the biochemical transformations of crotonates into butter and acetates [55]. In our study, A.crotonatoxidans was much more present in the supplemented groups than in control groups' sows.

Another important and often analysed group of microorganisms are the Lactobacillales, a diverse and phylogenetically heterogeneous order of lactic acid-producing bacteria that include the genus Lactobacillus, composed of over 170 species. They utilise carbohydrate fermentation and produce lactic acid as a major end-product [56]. Several species and strains are considered to be among the most promising probiotics involved in the modulation of gut microbiota and prevention of pathological stages, especially thanks to their protective role against inflammatory intestinal diseases and reconstitution of barrier defects. A few studies also demonstrated an improvement in the gastrointestinal barrier function by the proliferation of harmful bacteria [57]. However, it is postulated that transient colonisation is often a typical attribute of lactobacilli and their survival in the GI tract is highly variable among strains. Based on the several human studies, faecal prevalence of Lactobacilli shows fluctuations and a lack of stability [58]. Our study did not reveal the presence of Lactobacillus rhamnosus and Lactobacillus fermentum as being administered to sows orally in group B, supplemented with those probiotic composition. There are possibly two reasons for this: the attachment to the mucosa of the preceding initial sections (jejunum, duodenum) where they may manifest their beneficial properties [59] or both strains may have a low stability of the presence taking into account faecal samples. Any conclusion is complicated by the fact that sows were sampled only once, during delivery. Despite this fact, supplementation of the proposed probiotic's composition supports significantly the presence of the Lactobacillaceae in general taking into account the Family level and the Genus Lactobacillus compared with the control group of sows. Also, garlic extract improves participation of the above-mentioned microorganisms compared with the control group, although not as strongly as probiotics. As abundant inhabiting species in this study, we can consider Lactobacillus ultunensis only, because sequences characteristic for the remaining species (L. antri, pontis, reuteri) were not detected above $1 \%$ in 

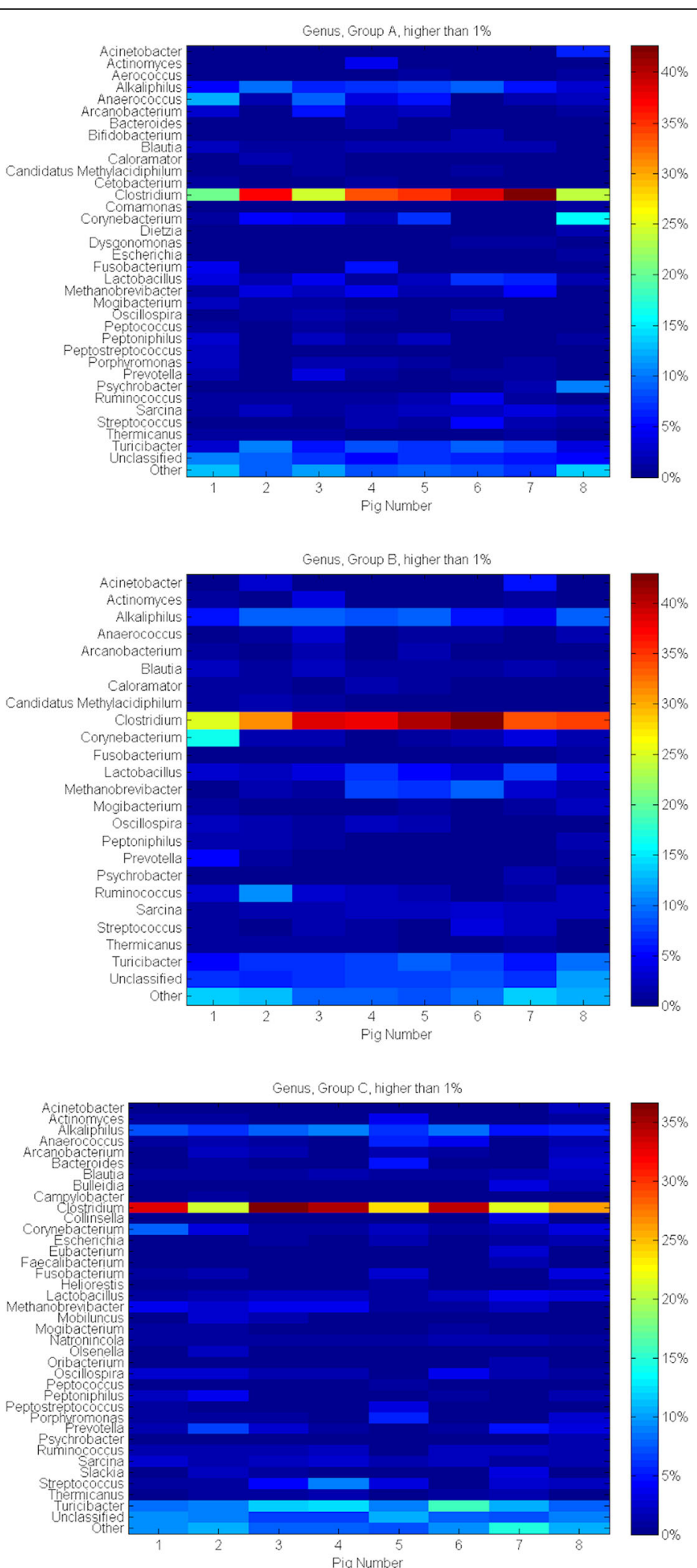

Fig. 6 (See legend on next page.) 
(See figure on previous page.)

Fig. 6 Prevalent Genera analysis heat-maps of pig faecal microbiota considering feed additives and individual animals. The heat-map shows the relative abundance of identified sequences between individuals within the examined groups on the Genus level. Density and saturation of each colour identify percentage (\%) of sequences specific for analysed taxonomic rank. Presented are only sequences exceeding 1\%. As 'Other' were marked the remaining sequences identified on the level lower than $1 \%$

all groups. Of the remaining species $L$. reuteri is widely described in the literature, as bacteria able to produce a variety of antimicrobial substances such as lactic acid, hydrogen peroxide, reuterin and reutericyclin, which exhibits beneficial effects for the organism. In the pig industry, the administration of $L$. reuteri resulted in an improvement in the growth rate, feed efficiency and reduction of diarrhoea incidence in neonatal and growing pigs [60].

In our study we also observed some tendencies taking into account non-abundant taxa $(<1 \%)$ of metabolic bacteria like Acinetobacter indicus, Bacteroides sartorii, Bifidobacterium pseudolongum, Cetobacterium ceti, Clostridium saccharoperbutylacetonicum, Corynebacterium hansenii, Psychrobacter halophilus and Psychrobacter proteolyticus. The majority of these bacterial species involved in metabolic changes in the intestinal lumen and supplementation of garlic extract seems to promote their participation in the microbiome composition. Bifidobacteria (among them Bifidobacterium pseudolongum) are generally considered to promote intestinal health by preventing the colonisation of potential pathogens: they reduce intestinal $\mathrm{pH}$ through an increased amount of fermentation products, produce inhibitory substances like bacteriocins and stimulate the immune system [61].

Analysis of the selected microbial function based on the literature data indicate that both additives used in this study (garlic extract and composition of probiotics) seems to create a greater metabolic potential than estimated in the control group of sows. Similarly, to other authors $[39,50,62]$ that confirmed some shifts in the gut microbiome after the supplementation of antibiotics, we estimated a similar tendency for supplemented probiotics as well as garlic extract. Importantly, proposed additives generate a selection of specific bacterial populations that contribute to the metabolism of animals. Further studies are needed to explain if estimated shifts in the composition of metabolic bacteria may also have a direct influence on the improvement of a pig's growth or fattening performance. It is also essential to understand whether or not the detected shapes of the microbiome after the addition of garlic or probiotic supplementation affect the cross-feeding relationship between individual members of the microbiome.

Another important aspect of our study was to find out if the supplementation of garlic extract, or a composition of probiotic bacteria, could modulate gut microbiome in such a way as to influence the typical pathogenic bacteria. Soler et al. [63] adopted colistin sulphate and amoxicillin to estimate the effect of supplemented microbials on the digestive microbiota. They analysed the influence of antibiotics starting with the higher taxonomic ranks of microbiome such as Phylum and have demonstrated a relative increase in Bacteroidetes in conjunction with a decrease in Firmicutes. In our study, phytobiotic properties of garlic extract as well as probiotics used in pigs seem to be oriented towards a decrease of Bacteroidetes. The proportion of Firmicutes in sows supplemented with garlic is slightly lower than in controlled pigs but we noted a significant increase in this group of microorganisms after probiotic supplementation. This does not, however, change the fact that in our study Gram-positive Firmicutes as well as Gram-negative Bacteroidetes represent a dominated Phila, described as being essential sign of healthy adult microbiota. Relation and proportion between this two Phyla (Bacteroidetes: Firmicutes) may contribute to disease states, however high variability in this proportion has been observed [64]. The relevance of the described ratio is therefore debatable in humans. In veterinary medicine, a lower $\mathrm{Bac}$ teroidetes: Firmicutes ratio was established for diarrheal piglets [65] but we did not observe any clinical disorders in the examined sows.

Administration of tested antimicrobials in those research studies seems to have an influence on Lactobacillus spp. depletion with the reduction of aerobic and anaerobic bacteria, especially Gram-negative bacteria. The researchers determined a decrease in the abundance of Proteobacteria and Lactobacillus in the antibiotictreated group and a shift in the abundance of the Family Prevotellaceae species, which cover the emerged niche [63]. In our study, garlic extract as well as the probiotics have the opposite impact on Lactobacillaceae. We observed an increase in the abundance of this Family in both of the supplemented groups. We also confirmed an opposite shift for the Family Prevotellaceae. Administration of both supplemented additives may have an effect on the Prevotellaceae reduction. In the analysis of the sequences identified within Family and Genus taxon rank, garlic extract as well as probiotics seem to reduce participation of Streptococci. On the Species level Streptococcus suis (etiological agent of streptococcosis, that can be isolated from the lungs, brain, heart and joints of infected animals) was confirmed in the controlled sows 


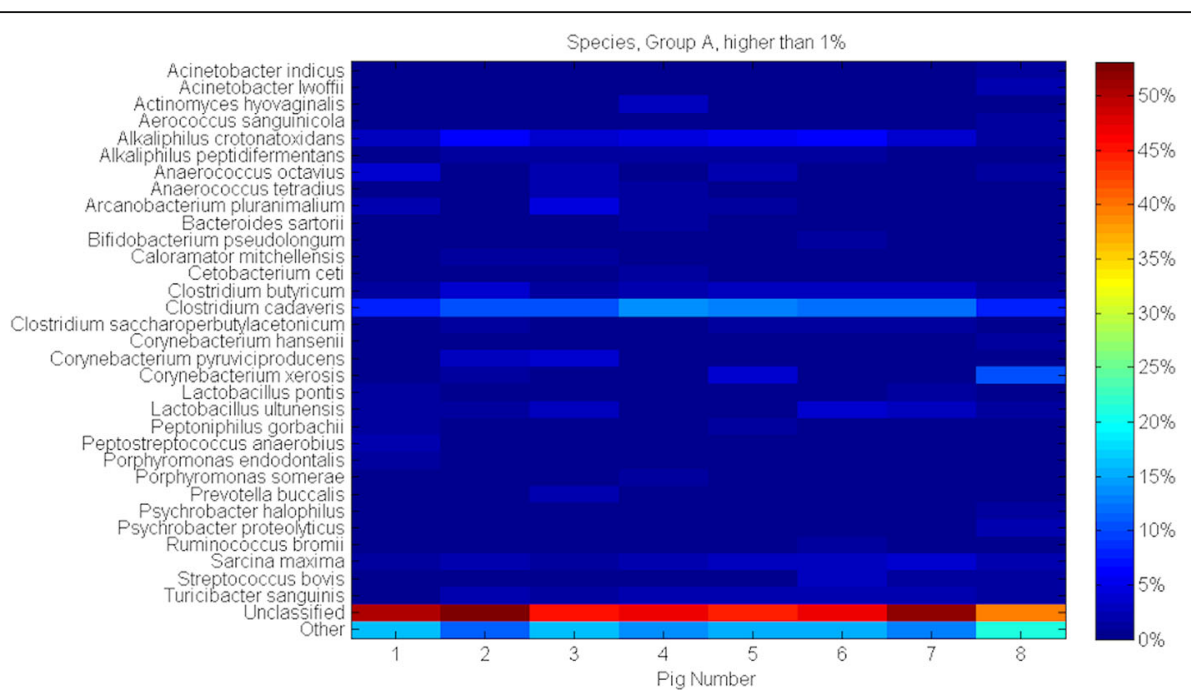

Species, Group B, higher than $1 \%$

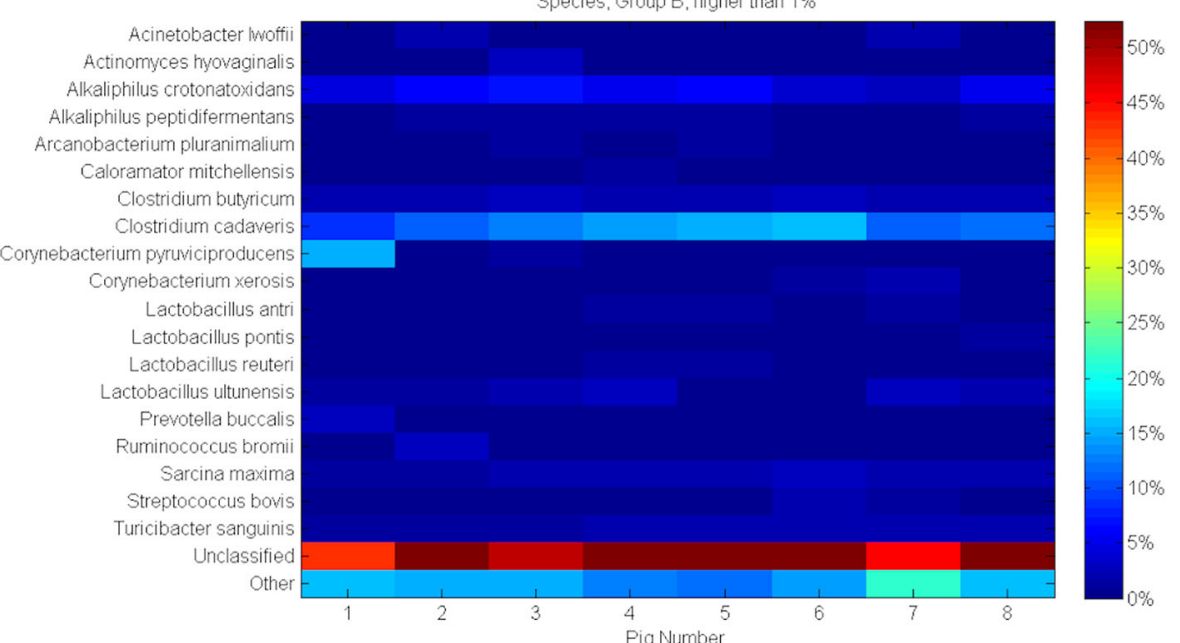

Species, Group C, higher than $1 \%$

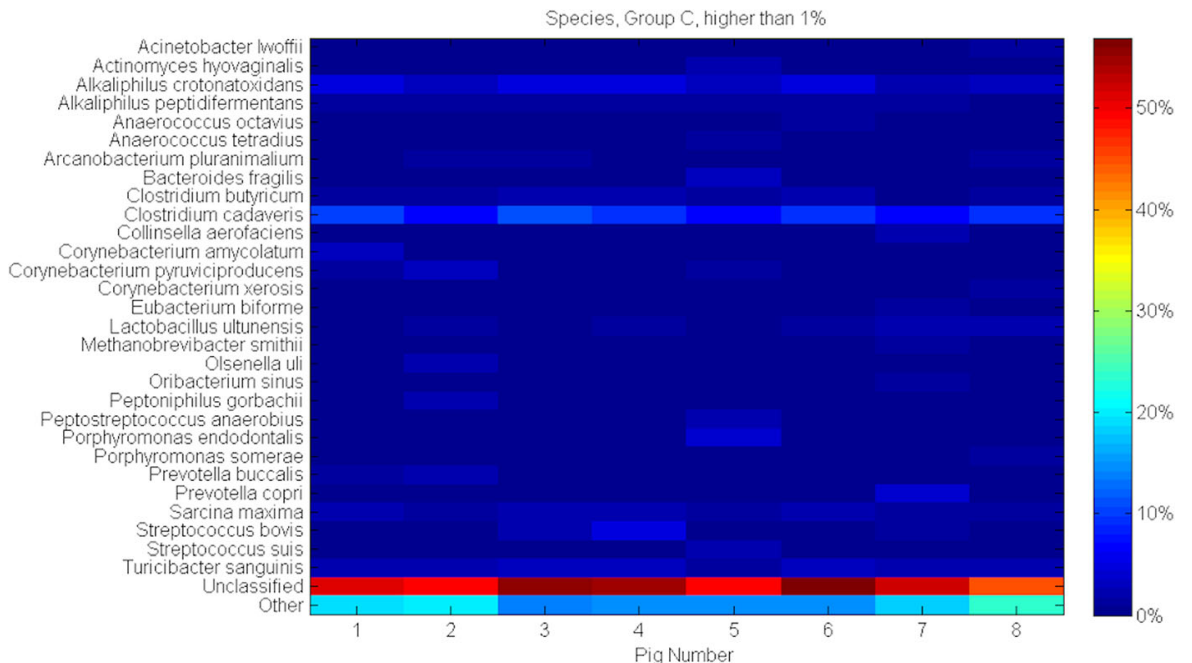

Fig. 7 (See legend on next page.) 
(See figure on previous page.)

Fig. 7 Prevalent Species analysis heat-maps of pig faecal microbiota considering feed additives and individual animals. The heat-map shows the relative abundance of identified sequences between individuals within the examined groups on the Species level. Density and saturation of each colour identify percentage (\%) of sequences specific for analysed taxonomic rank. Presented are only sequences exceeding 1\%. As 'Other' were marked the remaining sequences identified on the level lower than $1 \%$

(group C) only and was not identified in pigs supplemented with garlic or probiotics. Streptococcus suis is one of the most important pathogens in pigs causing arthritis, meningitis, septicaemia and many other infections, what is more it is a crucial zoonotic agent responsible for septicaemia, meningitis and other infections in humans [66]. So, in the prevention of streptococcal disease supplemented additives could be considered for future use. Actinomyces hyovaginalis, bacteria that may be associated in pigs with disseminated necrotic lung lesions, was identified as being non-abundant in all groups [67]. Out of the bacteria that may have an influence on an animal's health status, single papers describe Bacteroides fragilis, Colinsella aerofaciens, Corynebacterium amycolatum or Olsenella uli. Garlic extract as well as probiotics had an influence on the participation of Bacteroides fragilis, that was not identified in all animals from groups A and B. B. fragilis is considered to be an opportunistic pathogen, however it may exhibit both pathogenic and beneficial properties in the host [68]. In pigs, enterotoxigenic strains were isolated from the intestinal contents of animals with diarrhoea [69]. Additionally, both additives show the possibility of reduction in Colinsella aerofaciens occurrence. This bacteria can be linked to health disorders such as irritable bowel syndrome in humans but we did not find any information that would link its participation to similar disorders in pigs [70]. Corynebacterium constitute a part of the normal flora of animals while others are opportunistic bacteria. Described by Wendt et al. [71] Corynebacterium suis, associated with urinary disease in pigs, were not identified in our study. In this study, differences between groups were evident in the richness of the selected species like Corynebecterium amycolatum (absent from groups A and B) and C.hanseni (absent from group B) microorganisms that could be isolated from wounds and pus respectively [72, 73]. It is also noticeable that Corynobacterium xerosis, described as isolated mostly from subcutaneous lesions in swine, occurred in our study most frequently in sows fed with garlic extract [74]. In turn, in association with probiotics we noted a higher prevalence of Corynebacterium puruviciproducens, bacteria which exhibits immunomodulatory properties and can stimulate hosts humoral immune response to pathogenic microorganisms by adjusting the function of macrophage [75]. Olsenella uli was isolated from an inflamed human mouth and occasionally from the blood of humans with local oral or gastrointestinal infections [76] and in our own study bacteria participated in the microbiome of control sows only. From a clinical perspective Campylobacteraceae, particularly species like Campylobacter coli, C. jejuni or C. lari may have an epidemiological significance in pigs (diarrhoeal disease) as well as in humans [77]. In our study, Campylobacteraceae were detected on just the Family and Genus taxonomic rank and only covered controlled sows. However, we did not find sequences characteristic for pathogenic species in the following steps of taxonomic identification. The lowest frequency of Erysipelotrichaceae was confirmed in sows supplemented with garlic extract, however the disappearance of Genus Erysipelothrix was observed in all groups. We also did not identify typical swine pathogenic species Erysipelothrix rusiopathiae.

\section{Conclusion}

To conclude, a general trend of losing or decreasing members of pathogenic species in the swine microbiome seems to occur in relation to both supplemented additives. Our study strongly supports the hypothesis that the intestinal microbiome of sows may be heavily and favourably shaped by the supplementation of garlic extract and examined probiotic composition. Probiotics seem to boost both the presence of Archaea and the participation of Firmicutes. As a result of probiotics supplementation, the highest biodiversity was noted within detectable Lactobacillus species. Probiotics seem to influence also the highest proportion of Corynebacterium puruviciproducens (known for its immunomodulatory properties) and Ruminococcus spp. (identified as important microbial symbionts). Garlic extract appears to create the general highest biodiversity of the sows' microbiome on the Species level. Supplementation of garlic may also limit the presence of pathogenic bacteria, taking into account Erysipelotrichaceae.

Further research upon feed additives is still needed because diet ingredients as well as microbe-microbe interactions are factors modulating gut microbiome composition. Therefore, the domination of some species in parallel with the disappearance of others may constitute a risk of dysbiosis also in pig production.

\section{Methods}

The trial was performed on a private commercial pig farm, where the sow herd consisted of 130 animals. 
Farm owner has consented to the study. The feed for the sows was individually dosed using an electronic sow feeding system (Wolbrink NEDAP, Netherlands). The standard feed used for sows on the farm was as follows:

Sows before mating up to a week before farrowing (1 ton): $50 \%$ barley, $22.5 \%$ triticale, $20 \%$ rye, $5 \%$ soybeans, $2.5 \%$ premix* Polfamix LP TOP $1^{\text {a }}$ (Trouw Nutrition Polska Ltd.)

*a Polfamix LP TOP1: Lysine $4 \mathrm{~g}$, Methionine $2 \mathrm{~g}$, Threonine 2 g, Total Calcium 297 g, Total Phosphate 69 g, Sodium 62 g, Magnesium 10 g, Vitamin A 480000 IE, Vitamin D3 65,000 IE, Vitamin E $3000 \mathrm{mg}$, Vitamin K $80 \mathrm{mg}$, Vitamin B1 $80 \mathrm{mg}$, Vitamin B2 $200 \mathrm{mg}$, Vitamin B6 $150 \mathrm{mg}$, Vitamin B12 $1200 \mathrm{mcg}$, Biotin $8000 \mathrm{mcg}$, Niacin $1250 \mathrm{mg}$, Folic Acid $200 \mathrm{mg}$, Ca Pantothenate $600 \mathrm{mg}$, Choline 12,000 mg, Manganese $3100 \mathrm{mg}$, Zinc $5000 \mathrm{mg}$, Iron $4000 \mathrm{mg}$, Copper $990 \mathrm{mg}$, Iodine $30 \mathrm{mg}$, Selenium 12 mg, Betaine 5500 mg, Antioxidant 1000 mg.

Sows a week before delivery up to about a week after weaning piglets (1 ton): $50 \%$ barley, $30 \%$ wheat, $15 \%$ soybeans, premix* 4\% Polfamix LK TOP2 ${ }^{\mathrm{b}}$ (Trouw Nutrition Polska Ltd.) 0.7\% soybean oil, 0.3\% feed acidizer, JHJ Baracid (JHJ Sp. o.o.). In addition, after weaning - until next mating sows receive $5 \%$ glucose to feed (200 g / animal / day).

*b Polfamix LK TOP2: Lysine $50 \mathrm{~g}$, methionine $7 \mathrm{~g}$, Threonine $8 \mathrm{~g}$, Total calcium $230 \mathrm{~g}$, Total Phosphorus 60 g, Sodium 55 g, Magnesium 10 g, Vitamin A 300000 IE, Vitamin D3 50,000 IE, Vitamin E 3000 mg, Vitamin K 100 mg, Vitamin B1 55 mg, Vitamin B2 225 mg, Vitamin B6 $100 \mathrm{mg}$, Vitamin B12 $1000 \mathrm{mcg}$, Biotin 10,000 mcg, Niacin $700 \mathrm{mg}$, Folic Acid $50 \mathrm{mg}$, Ca Pantothenate $500 \mathrm{mg}$, Choline 12,000 mg, Manganese $2500 \mathrm{mg}$, Zinc $2500 \mathrm{mg}$, Iron $3000 \mathrm{mg}$, Copper $625 \mathrm{mg}$, Iodine $30 \mathrm{mg}$, Selenium $12 \mathrm{mg}$, Betaine $5500 \mathrm{mg}$.

On day 80 of the pregnancy twenty-four pregnant crossbred sows (Polish Landrase $x$ Polish Large White) were randomly selected and assigned to one of three groups: A $(n=8)$ - supplemented with garlic (Allium sativum) extract; B $(\mathrm{n}=8)$ - supplemented with probiotic bacteria; and $C(n=8)$ - the control group, without any additional supplementation. Only the negative control was included in the study. The positive control (sows supplemented with antibiotics) was not included in this research due to the EU-wide ban on the use of antibiotics as antimicrobial growth promoters (AGP) in animal feed (entered on January 1, 2006).

In group A the garlic extract additive (Allivet ${ }^{\mathrm{TM}}$, solution of natural garlic oil extract, level of analytical constituents: crude fat - $0.71 \%$, sodium $-24 \mathrm{mg} / \mathrm{kg}$; Centaur, Poland) was administered in a dose of $10 \mathrm{ml} /$ sow every 3 days, from the 80th day of gestation to the weaning day, according to the manufacturer's recommendation.
Probiotic bacteria Enterococcus faecium, Lactobacillus rhamnosus and Lactobacillus fermentum were administered in a dose of $2 \mathrm{~g} / \mathrm{sow} /$ day from the 80th day of gestation to the weaning day. The strains of probiotic bacteria Enterococcus faecium CCM 6226 (serial number: 030714), Lactobacillus rhamnosus CCM 1825 (serial number: 010914) and Lactobacillus fermentum CCM 7192 (serial number: 011014) were selected from a collection of microorganisms of PharmaGal-Bio (Slovakia) - all strains are admitted to trading as feed additives (EC Regulation 1831/2003) and are on the updated list from 24.10.2018 [78]. The mixture of probiotic bacteria was prepared integrating corn starch $(690 \mathrm{~g})$, maltodextrin $(50 \mathrm{~g})$ and protein $(250 \mathrm{~g})$ with $10 \mathrm{~g}$ of the probiotic bacteria $\left(1 \times 10^{9} \mathrm{CFU} / \mathrm{g}\right)$ in every $1 \mathrm{~kg}$. Both additives were fed individually by direct oral administration.

Due to the fact that the preparations are approved for use in animals, after the study sows remained for breeding use on the farm.

On the day of delivery (during farrowing), faecal swabs were collected for DNA extraction and sequencing analysis using sterile rectal swabs (Deltalab, Poland). DNA extraction and all procedures related to the new generation sequencing (NGS) analysis were performed in GENOMED S.A. (Warsaw, Poland). Genomic DNA from each sample was isolated using Genomic Mini AX Bacteria (A\&A Biotechnology, Poland) according to the manufacturer's instruction, with an additional mechanical lyses of each sample supported by zircon balls in a FastPrep ${ }^{\circledR}$ homogenizer (MP Biomedicals, Poland). Each swab was placed in a tube containing $500 \mu \mathrm{l}$ of the BS buffer for bacteria suspension, adding $20 \mu \mathrm{l}$ of lysozyme and $5 \mu \mathrm{l}$ of mutanolysin (lysozyme and mutanolysin provide a synergistic action by increasing the effectiveness of lysis of the bacterial cells from the Genera Streptococcus, Lactobacillus, Lactococcus, Listeria). Then samples were mixed and incubated in the Eppendorf TermoMixer $\left(50{ }^{\circ} \mathrm{C}, 20\right.$ min, 1400 RPM (revolutions per minute). The suspension $(400 \mu \mathrm{l})$ was transferred to centrifuge tubes and shaken with zirconia balls (60s, BeadBeater $\odot$, Biospec Products). Finally, $1 \mathrm{ml}$ of lysis buffer (LS) and $20 \mu \mathrm{l}$ of proteinase $\mathrm{K}$ were added.

Bacterial DNA was confirmed using real-time PCR in the thermocycler Stratagen Mx3000P (Thermofisher Scientific, USA) and SYBR Green (Sigma Aldrich, USA) as a fluorochrome. For the amplification of the fragment of bacterial gene $16 \mathrm{~S}$ rRNA, the universal primers have been used [79]. The PCR-mixture and the timetemperature profile were as below (Table 4).

Hypervariable regions V3-V4 of the $16 \mathrm{~S}$ rRNA gene were amplified. For amplification of the selected region and the preparation of the DNA libraries a pair of primers $341 \mathrm{~F}$ and $785 \mathrm{R}$ were used. The PCR reaction 
Table 4 Composition of the PCR mixture and temperature profile for the bacterial gene 16S rRNA amplification

\begin{tabular}{ll}
\hline Real Time 2x-PCR Mix SYBR A & $10 \mu \mathrm{l}$ \\
Starter 1055F $10 \mu \mathrm{M} 5^{\prime}$-ATGGCTGTCGTCAGCT-3' & $0.4 \mu \mathrm{l}$ \\
Starter 1392R $10 \mu \mathrm{M} 5^{\prime}$-ACGGGCGGTGTGTAC-3' & $0.4 \mu \mathrm{l}$ \\
DNA template & $1 \mu \mathrm{l}$ \\
deionized water & $8.2 \mu \mathrm{l}$ \\
initial denaturation & $95^{\circ} \mathrm{C}, 3$ \\
& $\mathrm{~min}$. \\
denaturation & $95^{\circ} \mathrm{C}, 15 \mathrm{~s}$ \\
priming & $58^{\circ} \mathrm{C}, 30 \mathrm{~s}$ \\
elongation & $72^{\circ} \mathrm{C}, 30 \mathrm{~s}$ \\
appointment of the melting curve (measurement of the & $65^{\circ} \mathrm{C}->$ \\
fluorescence in each temperature) & $95^{\circ} \mathrm{C}$
\end{tabular}

Abbreviations: PCR Polymerase chain reaction, PCR Mix SYBR A - ready-to-use mixture to the real-time PCR with fluorophore (A\&A Biotechnology, Poland); $F=$ forward primer; $R=$ reverse primer

was performed using NEBNext ${ }^{\oplus}$ Q5 Hotsart HighFidelity DNA Polymerase (NEB). Table 5 shows the time-temperature profile for the DNA libraries.

The new generation sequencing was performed in a MiSeq Reagent Kit v2 (Illumina) sequencer and the data analysis performed using MiSeq Reporter (MSR) v2.6 software and the $16 \mathrm{~S}$ Metagenomics protocol. The analysis consisted of three parts: the automatic demultiplexing of samples, the generation of fastq files containing raw readings and classification of reads (paired-end type) in the various taxonomic categories. The 16S Metagenomics protocol ensures species-specific classification of the obtained readings based on the reference sequences database Greengenes v13_5, modified by Illumina. Preparation of the reference database involved: filtering of the sequences shorter than $1250 \mathrm{bp}$, a filtering of the sequences containing more than 50 degenerated bases $(M$, $\mathrm{R}, \mathrm{W}, \mathrm{S}, \mathrm{Y}, \mathrm{K}, \mathrm{V}, \mathrm{H}, \mathrm{D}, \mathrm{B}, \mathrm{N}$ ); filtering the not fully classified sequences (the lack of classification on the taxonomic "genus" or "species" level). Taking into account that possible shifts in the intestinal microbiota after garlic or probiotic bacteria administration have been

Table 5 The time-temperature profile for metagenomic analysis of the $16 \mathrm{~S}$ rRNA V3-V4 hypervariable region

\begin{tabular}{llll}
\hline NGS PCR profile & & & \\
\hline Step & Temperature & Time & No. of cycles \\
\hline initial denaturation & $98^{\circ} \mathrm{C}$ & $30 \mathrm{~s}$ & 1 \\
denaturation & $98^{\circ} \mathrm{C}$ & $10 \mathrm{~s}$ & $3-15$ \\
annealing & $65^{\circ} \mathrm{C}$ & $75 \mathrm{~s}$ & \\
final extension & $65^{\circ} \mathrm{C}$ & $5 \mathrm{~min}$ & 1 \\
final hold & $4-10^{\circ} \mathrm{C}$ & $\infty$ &
\end{tabular}

Abbreviations: 16S rRNA 16S ribosomal RNA, NGS Next generation sequencing, $16 S$ V3-V4 Regions of interest-specific primer examined, data analysis obtained from the NGS was performed, including protocols with all sequences after filtering.

The diversity of microbial communities within groups $\mathrm{A}, \mathrm{B}, \mathrm{C}$ and between groups using sequence reads was analysed using two standard diversity measures: Shannon and Simpson diversity indices [80].

For a given community the Shannon diversity index is defined as

$$
D_{S h}=\exp \left(-\sum_{i=1}^{R} p_{i} \ln p_{i}\right)
$$

Here $R$ is the richness of the community (i.e. the total number of types in the community), whereas $p_{i}$ is the proportional abundance of the $i$-th type. $D_{S h}$ is the exponent of the Shannon entropy and it measures the level of uncertainty in the community. $D_{S h}$ ranges from 0 (when all the individuals are of the same type) to $R$ (when all $p_{i}=1 / R$ ).

The Simpson diversity index is defined as:

$$
D_{S i}=\frac{1}{\sum_{i=1}^{R} p_{i}^{2}}
$$

It measures the degree of concentration of the community (more precisely, it is the reciprocal of the probability that two individuals taken at random from the community are of the same type). Both diversity indices were calculated for all eight pigs from each group A, B and $\mathrm{C}$. The calculations were done separately on each available taxonomic level (Family, Genus and Species). All the calculations were performed in the MATLAB R2016a environment. The obtained results allowed us to compare the microbial diversity between the analysed groups.

\section{Abbreviations}

ADFI: Average daily feed intake; ADG: Average daily gain; AGP: Antibiotic growth promoter; bp: Base pair; BS buffer: Bacterial suspension buffer; DNA: Deoxyribonucleic acid; EC: European Commission; EU: European Union; FCR: Feed conversion ratio; Gl tract: Gastrointestinal tract; IgA: Immunoglobulin A; IgG: Immunoglobulin G; IgM: Immunoglobulin M; LS buffer: Lysis buffer; mRNA: Messenger ribonucleic acid; MSR: MiSeq Reporter; NEB: NEBNext ${ }^{\oplus}$ Q5 Hotsart High-Fidelity DNA Polymerase; NGS: Next generation sequencing; NK cells: Natural killer cells; PCR: Polymerase chain reaction; PFA: Phytogenic feed additive; QPC: Qualified Presumption of Safety; rRNA: Ribosomal ribonucleic acid; RPM: Revolutions per minute; SD: Standard deviation; TLR: Toll-like receptors

\section{Acknowledgements}

We thank the owner of the farm as well as Andrzej Giedrojć DVM and Andrzej Rudy DVM, PhD for technical support and assistance during sample collection. The research of MM was partially supported by NCN-DFG Beethoven Grant No.2016/23/G/ST1/04083. The research is financed under the Leading Research Groups support project from the subsidy increased for period 2020-2025 in the amount of 2\% of the subsidy reffered to Art. 387 (3) of the Law of 20 July 2018 on Higher Education and Sciences, obtained in 2019. 


\section{Authors' contributions}

MS and KPJ organized the project, planned the study and agreed the objectives thereof. MS selected the farm and animals, then organized and executed the sample collection. MM and ARz performed statistical analysis of the data. MS, KPJ, ARz and MM wrote the initial draft of the manuscript. KR made the critical revision and refinement of the manuscript. MS guaranteed the project's funding. KPJ and KR guaranteed funding of the proofreading. The authors read and approved the final manuscript.

\section{Funding}

The funding body was represented by one of the Authors (MS), who has lifelong specific competence in the present research field in farm conditions (swine diseases) and participated to the study design, samples collection, data interpretation, and final manuscript preparation and approval. In particular, the funding body patronized the study and collaborated with the farmer.

\section{Availability of data and materials}

Raw data for calculation of tables and figures are available from the corresponding author upon request.

\section{Ethics approval and consent to participate}

The study was conducted with ethical approval by the local authorities, the Local Ethics Committee for Animal Experiments at the University of Environmental and Life Sciences, Wroclaw, Poland (filed under No. 22/2014). Written informed consent to use the animals in the study was obtained from the owner of the animals (available from the corresponding author upon request). The Farmer was not forced to participate in the survey nor donate samples from animals. Name, region and the village of the farm was registered.

After the study all sows remained on the farm for breeding use (as before the start of the research).

\section{Consent for publication}

Not applicable.

\section{Competing interests}

The authors declare that they have no competing interests.

\section{Author details}

'Department of Epizootiology with Clinic for Birds and Exotic Animals, Faculty of Veterinary Medicine, Wrocław University of Environmental and Life Sciences, Plac Grunwaldzki 45, Wrocław, Poland. ${ }^{2}$ Hugo Steinhaus Center, Faculty of Pure and Applied Mathematics, Wrocław University of Science and Technology, Wyspianskiego 27, Wrocław, Poland. ${ }^{3}$ Department of Immunology, Pathophysiology and Veterinary Preventive Medicine, Faculty of Veterinary Medicine, Wrocław University of Environmental and Life Sciences, Norwida 31, Wrocław, Poland.

\section{Received: 30 October 2019 Accepted: 30 October 2020} Published online: 13 November 2020

\section{References}

1. Isaacson R, Kim HB. The intestinal microbiome of pigs. Anim Health Res Rev. 2012;13:100-9

2. Kim HB, Borewicz K, White BA, Singer RS, Sreevatsan S, Tu ZJ, et al. Longitudinal investigation of the age-related bacterial diversity in the feces of commercial pigs. Vet Microbiol. 2011;153:124-33.

3. Niederwerder MC. Role of microbiome in swine respiratory disease. Vet Microbiol. 2017;209:97-106.

4. Han GG, Lee JY, Jin GD, Park J, Choi YH, Kang SK, et al. Tracing of the fecal microbiota of commercial pigs at five growth stages from birth to shipment. Sci Rep. 2018;8:6012. https://doi.org/10.1038/s41598-018-24508-7.

5. Holman DB, Brunelle BW, Trachsel J, Allen HK. Meta-analysis to define a core microbiota in the swine gut. mSystems. 2017;2(3):e00004-17. https://doi. org/10.1128/mSystems.00004-17.

6. Urubschurov V, Janczyk P, Souffrant WB, Freyer G, Zeyner A. Establishment of intestinal microbiota with focus on yeasts of unweaned and weaned piglets kept under different farm conditions. FEMS Microbiol Ecol. 2011;77: 493-502.
7. Shan T, Li L, Simmonds P, Wang C, Moeser A, Delwart E. The fecal virome of pigs on a high-density farm. J Virol. 2011;85:11697-708.

8. Castanon JIR. History of the use of antibiotics as growth promoters in European poultry feeds. Poult Sci. 2007;86:2466-71.

9. Millet $\mathrm{S}$, Maertens $\mathrm{S}$. The European ban on antibiotic growth promoters in animal feed: from challenges to opportunities. Vet J. 2011;187:143-4.

10. An official website of the European Union. (https://ec.europa.eu) Accessed on 24 Oct 2018.

11. Shim SB, Verstegen MW, Kim IH, Kwon OS, Verdonk JM. Effects of feeding antibiotic-free creep feed supplemented with oligofructose, probiotics or synbiotics to suckling piglets increases the preweaning weight gain and composition of intestinal microbiota. Arch Anim Nutr. 2005;59(6):419-27.

12. Jacela JY, DeRouchey JM, Tokach MD, Goodband RD, Nellsen JL, Renter DG, et al. Feed additives for swine: fact sheets - acidifiers and antibiotics. J Swine Health Prod. 2009;17:270-5.

13. Szuba-Trznadel A, Rząsa A, Lira R, Fuchs B. The influence of $(1,3)-(1,6)-B-D-$ glucan on the production results of sows and their offspring. J Anim Feed Sci. 2014;23(3):228-35.

14. Liu Y, Espinosa CD, Abeilla JJ, Casas GA, Lagos LV, Kwon WB, et al. Non-antibiotic feed additives in diets for pigs - a review. Anim Nutr. 2018:4:113-25.

15. Hanczakowska E, Swiatkiewicz M. Effect of herbal extracts on piglet performance and small intestinal epithelial villi. Czeh J Anim Sci. 2012;57(9): 420-9.

16. Mehdi $Y$, Letourneau-Montminy MP, Goucher ML, Chorfi Y, Suresh G, Rouissi $T$, et al. Use of antibiotics in broiler production: global impacts and alternatives. Anim Nutr. 2018;4(2):170-8.

17. Tatara MR, Sliwa E, Dudek K, Gawron A, Piersiak T, Dobrowolski P, et al. Aged garlic extract and allicin improve performance and gastrointestinal tract development of piglets reared in artificial sow. Ann Agric Environ Med. 2008;15:63-9.

18. Lee DH, Ra CS, Song YH, Sung Kl, Kim JD. Effects of dietary garlic extract on growth, feed utilization and whole body composition of juvenile Sterlet sturgeon (Acipenser ruthenus). Asian Australas J Anim Sci. 2012;25(4):577-83. https://doi.org/10.5713/ajas.2012.12012.

19. Cheng G, Hao H, Xie S, Wang X, Dai M, Huang L, et al. Antibiotic alternatives: the substitution of antibiotics in animal husbandry? Front Microbiol. 2014. https://doi.org/10.3389/fmicb.2014.00217.

20. Noman ZA, Hasan MM, Talukder S, Sarker YA, Paul TK, Sikder MH. Effect of garlic extract on growth, carcass characteristics and haematological parameters in broilers. Bangladesh Vet. 2015;32(1):1-6.

21. Senthilkumar $S$, Madesh $N$, Purushothaman MR, Vasanthakumar $P$, Thirumalaisamy G, Sasikumar P. Effect of garlic supplementation on performance in broilers - a review. Int J Sci Environ Technol. 2015;4: 980-3.

22. Brzóska F, Śliwiński B, Michalik-Rutkowska O, Śliwa J. The effect of garlic (allium sativum $\mathrm{L}$ ) on growth performance, mortality rate, meat and blood parameters in broilers. Ann Anim Sci. 2015;15(4):961-75.

23. Sheoran N, Kumar R, Kumar A, Batra K, Sihag S, Maan S, et al. Nutrigenomic evaluation of garlic (Allium sativum) and holy basil (Ocimum sanctum) leaf powder supplementation on growth performance and immune characteristics in broilers. Veterinary World. 2017;10(1):121-9.

24. Liu Y, Che TM, Song M, Lee JJ, Almeida JA, Bravo D, et al. Dietary plant extracts improve immune response and growth efficiency of pigs experimentally infected with porcine reproductive and respiratory syndrome virus. J Anim Sci. 2013;91(12):5668-79.

25. Yan L, Meng QW, Kim IH. Effects of fermented garlic powder supplementation on growth performance, nutrient digestibility, blood characteristics and meat quality in growing-finishing pigs. Anim Sci J. 2012; 85(5):411-7. https://doi.org/10.1111/j.1740-0929.2011.00973.x.

26. Adebiyi OA, Ajayi OS, Adejumo 10, Osungade TO. Performance, microbial load and gut morphology of weaned pigs fed diets supplemented with turmeric, ginger and garlic extracts. Trop Anim Prod Invest. 2014;17(1):25-31.

27. Estienne MJ, Harstock TG, Harper AF. Effects of antibiotics and probiotics on suckling pig and weaned pig performance. Int J Appl Res Vet Med. 2005; 3(4):303-8.

28. Szabó I, Wieler LH, Tedin K, Scharek-Tedin L, Taras D, Hensel A, et al. Influence of a probiotic strain of enterococcus faecium on salmonella enterica serovar Typhimurium DT104 infection in a porcine animal infection model. Appl Environ Microbiol. 2009;96:219-33. 
29. Liao SF, Nyachoti M. Using probiotics to improve swine gut health and nutrient utilization. Anim Nutr. 2017;3(4):331-43.

30. Markowiak $P$, Śliżewska K. The role of probiotics, prebiotics and synbiotics in animal nutrition. Gut Pathogens. 2018;10:21. https://doi.org/10.1186/ 2Fs13099-018-0250-0.

31. De Cupere F, Deprez P, Demeulenaere D, Muylle E. Evaluation of the effect of 3 probiotics on experimental Escherichia coli enterotoxaemia in weaned piglets. J Vet Med B. 1992;39:277-84.

32. Kreuzer S, Janczyk P, Assmus J, Schmidt MFG, Brockmann GA, Nöckler K. No beneficial effects evident for enterococcus faecium NCIMB 10415 in weaned pigs infected with salmonella enterica serovar Typhimurium DT104. Appl Environ Microbiol. 2012;78:4816-25.

33. Dowarah $R$, Agarwal AKVN. The use of lactobacillus as an alternative of antibiotic growth promoters in pigs. Anim Nutr. 2017;3(1):1-6. https://doi. org/10.1016/j.aninu.2016.11.002.

34. Napiórkowska B, Dobrowolska Z, Więcek J, Gajewska J, Rekiel A. Effect of a probiotic preparation on daily weight gain, survival rate and composition of faecal microflora in piglets (in Polish). Roczniki Naukowe Polskiego Towarzystwa Zootechnicznego. 2014;10(1):57-68.

35. Zeyner A, Boldt E. Effects of probiotic Enterococcus faecium strain suplemmented from birth to weaning on diarrhoea patterns and performance of piglets. J Anim Physiol Anim Nutr. 2006;90(1-2):25-31.

36. Brzozowski B, Bednarski W, Gołek P. The adhesive capability of two lactobacillus strains and physicochemical properties of their synthesized biosurfactants. Food Technol Biotechnol. 2011:49(2):177-86.

37. Zhang L, Xu Y, Liu H, Lai T, Ma J, Wang J, et al. Evaluation of Lactobacillus rhamnosus GG using an Escherichia coli K88 model of piglet diarrhoea: effects on diarrhoea incidence, faecal microflora and immune responses. Vet Microbiol. 2010;141(1-2):142-8.

38. Cai CJ, Cai PP, Hou CL, Zeng XF, Qiao SY. Administration of Lactobacillus fermentum 15007 to young piglets improved their health and growth. J Anim Feed Sci. 2014;23(3):222-7.

39. Kim HB, Borewicz K, Whiteb BA, Singera RS, Sreevatsana S, Tuc ZJ, et al. Microbial shifts in the swine distal gut in response to the treatment with antimicrobial growth promoter, tylosin. PNAS. 2012;109(38):15485-90.

40. McCormack UM, Curião T, Wilkinson T, Metzler-Zebeli BU, Reyer H, Ryan T, et al. Fecal microbiota transplantation in gestating sows and neonatal offspring alters lifetime intestinal microbiota and growth in offspring. mSystems. 2018;3(3):e00134-17. https://doi.org/10.1128/mSystems.00134-17.

41. Quan J, Cai G, Ye J, Yang M, Ding R, Wang $X$, et al. A global comparison of the microbiome compositions of three gut locations in commercial pigs with extreme feed conversion ratios. Sci Rep. 2018;8:4536. https://doi.org/10. 1038/s41598-018-22692-0.

42. Brässen C, Esser D, Rauch B, Siebers B. Carbohydrate metabolism in Archaea. Current insights into unusuall enzymes and pathways and their regulation. Microbiol Mol Biol Rev. 2014;78(1):89-175.

43. Thauer RK, Kaster AK, Seedorf H, Buckel W, Hedderich L. Methanogenic archaea: ecologically relevant differences in energy conservation. Nat Rev Microbiol. 2008;6(8):579-91.

44. Jabłoński S, Rodowicz P, Łukaszdewicz M. Methanogenic Archaea database containing physiological and biochemical characteristics. Int J Syst Evol Microbiol. 2015;65:1360-8.

45. Brugère JF, Borrel G, Gaci N, Tottey W, O'Tole PW, Malpuech-Brugère C. Archaebiotics: proposed therapeutic use of archaea to prevent trimethylaminuria and cardiovascular disease. Gut Microbes. 2014:5(1):5-10. https://doi.org/10.4161/gmic.26749.

46. Brugère JF, Ben Hania W, Arnal ME, Ribière C, Ballet N, Vandeckerkove $P$ et al. Archaea: microbial candidates in next generation probiotics development. J Clin Gastroenterol. 2017;52(Suppl 1):S71-3. https://doi.org/ 10.1097/MCG.0000000000001043 Proceedings from the 9th Probiotics, Prebiotics and New Foods, Nutraceuticals and Botanicals for Nutrition \& Human and Microbiota Health Meeting: 10 to 12 September 2017; Rome.

47. La Reau AJ, Meier-Kolthoff JP, Suen G. Sequence-based analysis of the genus Ruminococcus resolves its phylogeny and reveals strong host association. Microbial Genomics. 2016;2. https://doi.org/10.1099/mgen.0. 000099

48. Ze X, Duncan SH, Louis P, Flint HJ. Ruminococcus bromii is a keystone species for the degradation of resistant starch in human colon. ISME J. 2012; 6:1535-43.

49. Kovatcheva-Datchary P, Nillson A, Akrami R, Lee YS, De Vadder F, Arora T, et al. Dietetary fiber-induced improvement in glucose metabolism is associated with increased abundance of Prevotella. Cell Metab. 2015;22:97182.

50. Frese SA, Parker K, Calvert CC, Mills DA. Diet shapes the gut microbiome of pigs during nursing and weaning. Microbiome. 2015;3(28). https://doi.org/ 10.1186/s40168-015-0091-8.

51. Flint HJ, Bayer EA, Rincon MT, Lamed R, White BA. Polysaccharide utilization by gut bacteria: potential for new insights from genomic analysis. Nat Rev Microbiol. 2008;6:121-31.

52. Vital M, Howe AC, Tjedje JM. Revealing the bacterial butyrate synthesis pathways by analyzing meta (genomic) data. mBio. 2014;5(2):e00889. https://doi.org/10.1128/mBio.00889-14.

53. Cassir N, Benamar B, La Scola B. Clostridium butyricum: from benefitial to a new emerging pathogen. Clin Microbiol Infect. 2016;22:37-45.

54. Poduval RD, Mohandas R, Unnikrishnan D, Corpuz M. Clostridium Cadaveris Bactereia in an Immunocompetent host. Clin Infect Dis. 1999;29(1):1354-5.

55. Xianhua C, Xiaoli L, Xsiuzhu D. Alcaliphilus crotonatoxidans sp. nov., a strictly anaerobic, crotonate-dysmutating bacterium isolated from a methanogenic environment. Int J Syst Evol Microbiol. 2003;53(4):971-5.

56. Goldstein EJC, Tyrrell KL, Citron DM. Lactobacillus species: taxonomic complexity and controversial susceptibilities. Clin Infect Dis. 2015;60(2):98107.

57. Azad AK, Sarker M, Li T, Yin J. Probiotic species in the modulation of gut microbiota, an overview. Biomed Res Int. 2018. https://doi.org/10.1155/ 2018/9478630

58. Walter J. Ecological role of lactobacilli in the gastrointestinal tract: implication for fundamental and biomedical research. Appl Environ Microbiol. 2018;74(16):4985-96.

59. Govender M, Choonara YE, Kumar P, du Toit LC, van Vuuren S, Pillay V. A review of the advancements in probiotic delivery: conventional vs. nonconventional formulations for intestinal flora supplementation. J Am Assoc Pharmaceut Scientists. 2013;15(1):29-43.

60. Hou C, Zeng X, Yang F, Liu H, Qiao S. Study and use of the probiotic lactobacillus reuteri in pigs: a review. J Anim Sci Biotechnol. 2015;6(1):14.

61. Vasquez N, Suau A, Magne F, Pochart P, Pélissier M. Differential effects of Bifidobacterium pseudolongum strain Patronus and metronidazole in the rat gut. Physiol Biotechnol. 2009;75(2):381-6.

62. Looft T, Johnsonb TA, Allena HK, Baylesa DO, Alta DP, Stedtfeld RD, et al. Infeed antibiotic effects on the swine intestinal microbiome. PNAS. 2012; 109(5):1691-6.

63. Soler C, Goossens T, Bermejo A, Migura-Garcia L, Cusco A, Francino O, et al. Digestive microbiota is different in pigs receiving antimicrobials or feed additive during the nursery period. PLoS One. 2016;13(5):e0197353. https:// doi.org/10.1371/journal.pone.0197353.

64. Wexler AG, Goodman AL. An insider's perspective: Bacteroides as a window into the microbiome. Nat Microbiol. 2017;2:17026. https://doi.org/10.1038/ nmicrobiol.2017.26.

65. Bin P, Tang Z, Lju S, Chen S, Xia Y, Liu J, et al. Intestinal microbiota mediates Enterotoxigenic Escherichia coli-induced diarrhea in piglets. BMC Vet Res. 2018;14:385. https://doi.org/10.1186/s.12917-018-1704-9.

66. Goyette-Desjardins G, Auger JP, Xu J, Segura M, Gottschalk M. Streptococcus suis, an important pig pathogen and emerging zoonotic agent-an update on the worldwide distribution based on serotyping and sequence typing. Emerg Microbes Infect. 2014;3:6. https://doi.org/ 10.1038/emi.2014.45.

67. Aalbaeck B, Christensen $\mathrm{H}$, Bisqaard M, Lijegren $\mathrm{CH}$, Nielsen $\mathrm{OL}$, Jensen HE. Actinomyces hyovaginalis associated with disseminated necrotic lung lesions in slaughter pigs. J Comp Pathol. 2003;129(1):70-7.

68. Deng H, Li Z, Tan Y, Guo Z, Liu Y, Wang Y, et al. A novel strain of Bacteroides fragilis enhances phagocytosis and polarises M1 macrophages. Sci Rep. 2016;6:1-11.

69. Myers LL, Shoop DS. Association of enterotoxigenic Bacteroides fragilis with diarrheal disease in young pigs. Am J Vet Res. 1987;48(5):774-5.

70. Bag S, Ghosh TS, Dascorresponding B. Complete genome sequence of Collinsella aerofaciens isolated from the gut of a healthy Indian subject. Genome Announc. 2017;5(47):e01361-17.

71. Wendt M, Liebhold M, Kaup F, Amtsberg G, Bollwahn W. Corynebacterium suis infection in swine 1, clinical diagnosis with special considereation of urine studies and cystoscopy. Tieraerztliche Praxix. 1990;18(4):353-7.

72. Bernard K. The genus corynebacterium and other medically relevant coryneform-like bacteria. J Clin Microbiol. 2012. https://doi.org/10.1128/JCM. 00792-12. 
73. Sengupta M, Naina P, Balaji V, Anandan S. Corynebacterium amycolatum: an unexpected pathogen in the ear. J Clin Diagn Res. 2015;9(12):DD01-3.

74. Hernandez-Leon F, Acosta-Dibarrat J, Vasquez-Chagoyan JC, Fernandes Rosas P. Montes de Oca-Jimenez R. Identification and molecular

characterization of Corynebacterium xerosis isolated from a sheep cutaneous abscess: first case report in Mexico. BMC Res Notes. 2016;9:358. https://doi. org/10.1186/s13104-016-2170-8.

75. Tong J, Han Q, Wang S, Su Z, Zheng D, Shen P, et al. Corynebacterium pyruviciproducens, as an immune modulator, can promote the activity of macrophages and up-regulate antibody response to particulate antigen. Exp Biol Med. 2012;237(11):1322-30

76. Kraatz M, Wallace RJ, Svensson L. Olsenella umbonata sp. nov., a microaerotolerant anaerobic lactic acid bacterium from the sheep rumen and pig jejunum, and emended descriptions of Olsenella, Olsenella uli and Olsenella profusa. Int I Syst Evol Microbiol. 2011;61:795-803.

77. Modolo JR, Margato LFF, Gottschalk AF, de Magalhaes Lopez CA. Incidence of campylobacter in pigs with and without diarrhea. Rev Microbiol. 1999;30: 19-21.

78. An official website of the European Union [https://ec.europa.eu/food/sites/ food/files/safety/docs/animal-feed-eu-reg-comm_register_feed_additives_1 831-03.pdf.] Accessed on 24 Oct 2018.

79. Ferris MJ, Muyzer G, Ward DM. Denaturing gradient gel electrophoresis profiles of 165 rRNA-defined populations inhabiting a hot spring microbial mat community. Appl Environ Microbiol. 1996;62:340-6.

80. Jost L. Entropy and diversity. OIKOS. 2006;113(2):363-75.

\section{Publisher's Note}

Springer Nature remains neutral with regard to jurisdictional claims in published maps and institutional affiliations.

Ready to submit your research? Choose BMC and benefit from:

- fast, convenient online submission

- thorough peer review by experienced researchers in your field

- rapid publication on acceptance

- support for research data, including large and complex data types

- gold Open Access which fosters wider collaboration and increased citations

- maximum visibility for your research: over $100 \mathrm{M}$ website views per year

At $\mathrm{BMC}$, research is always in progress.

Learn more biomedcentral.com/submissions 